PROCEEDINGS OF THE

AMERICAN MATHEMATICAL SOCIETY

Volume 133, Number 8, Pages 2297-2308

S 0002-9939(05)08067-6

Article electronically published on March 21, 2005

\title{
PIECEWISE LINEAR SPECTRAL SEQUENCES
}

\author{
YOUMING LIU AND YUESHENG XU
}

(Communicated by David R. Larson)

\begin{abstract}
We study a class of orthonormal exponential bases for the space $L^{2}[0,1]$ and introduce the concept of spectral sequences. We characterize piecewise linear spectral sequences with the knot at $1 / 2$ and investigate the non-continuity of the piecewise linear spectral sequences. From a special construction of a piecewise constant spectral sequence, the classical Walsh system is recovered.
\end{abstract}

\section{INTRODUCTION}

The classical Fourier functions $\mathcal{F}:=\left\{e_{n}: n \in Z\right\}$, where $Z:=\{\ldots,-1,0,1, \ldots\}$ and $e_{n}(t):=e^{2 \pi i n t}, t \in I:=[0,1]$, form an orthonormal basis for the space $L^{2}(I)$. This basis plays an important role in many areas of mathematics and engineering. However, since the Fourier basis functions have constant frequencies, it has serious limitations in some applications. In particular, when a signal is non-linear and nonstationary, the classical Fourier basis does not give a satisfactory representation of such a signal [4]. Finding a better basis has been a main research subject in harmonic analysis, approximation theory and signal analysis, such as window Fourier bases, Gabor bases, wavelet bases (cf., 2, 3, 6, 10) and intrinsic mode functions recently studied in 1 4.

The main purpose of this paper is to study a class of orthonormal exponential bases having non-constant frequencies. Specifically, we develop orthonormal bases

$$
f_{n}:=e^{2 \pi i g_{n}}, \quad n \in Z,
$$

for $L^{2}(I)$, where the frequency functions $g_{n}$ are piecewise linear on $I$. We anticipate that the non-constant frequency functions $g_{n}$ will allow us to better represent certain signals. In fact, as we will show in the last section of this paper, when $g_{n}$ are chosen as some piecewise constant functions, the basis $f_{n}, n \in Z$, turns out to be the well-known Walsh system (cf., 8) ). In other words, the classical Walsh system is an orthonormal exponential basis having piecewise constant frequency functions.

Received by the editors September 11, 2003.

2000 Mathematics Subject Classification. Primary 42C15.

Key words and phrases. Orthonormal exponential bases, non-constant frequency, spectral sequences, Walsh systems.

The first author was supported in part by the Natural Science Foundation of Beijing, No. 1022002 .

The second author was supported in part by the US National Science Foundation under grants 9973427 and 0312113, by the Natural Science Foundation of China under grant 10371122 and by the Chinese Academy of Sciences under the program "One Hundred Distinguished Chinese Young Scientists". 
Therefore, these bases unify the classical Fourier basis and the Walsh system, as well as many other interesting bases.

We now introduce the concept of spectral sequences. A sequence of real-valued functions $g_{n}, n \in Z$, defined on $I$, is called a spectral sequence of $I$ if the exponential function system $f_{n}, n \in Z$, defined by (1.1) in terms of $g_{n}$ is an orthonormal basis for $L^{2}(I)$. In some sense, the notion of spectral sequences is a generalization of the spectral sets, which attracted significant attention in recent years (see, for example, [5, 9] and the references cited therein).

We organize this paper into five sections. In Section 2, we describe two classes of piecewise linear spectral sequences. We establish in Section 3 characterizations of piecewise linear spectral sequences with the knot at $1 / 2$. Can we construct continuous non-trivial piecewise linear spectral sequences? We answer this question in Section 4 by providing a negative result. In Section 5, we construct a special piecewise constant spectral sequence and identify it with the classical Walsh system.

\section{Two COnstructions}

We describe two constructions of piecewise linear spectral sequences $g_{n}$ with the knot at $1 / 2$. We first present a simple technical lemma that will be used frequently in this paper.

Lemma 2.1. Let $c \in R$ be a fixed number. For any $a \in Z$, let $I(a, c):=$ $\int_{c}^{c+\frac{1}{2}} e^{2 \pi i a t} d t$. Then, the integral $I(a, c)=0$ if and only if $a \in 2 Z \backslash\{0\}$.

Proof. A direct computation confirms that $I(a, c)=\frac{e^{2 \pi i a c}}{2 \pi i a}\left(e^{\pi i a}-1\right)$, for $a \neq 0$, from which this lemma follows.

Throughout this paper, we are interested in frequency functions having the form

$$
g_{n}(t)= \begin{cases}a_{n} t+b_{n}, & t \in[0, \theta), \\ c_{n} t+d_{n}, & t \in[\theta, 1],\end{cases}
$$

where $a_{n}, b_{n}, c_{n}, d_{n} \in R$ and $\theta \in(0,1)$. These frequency functions define basis functions $f_{n}$ by equation (1.1).

Next, we present two classes of spectral sequences having the form given by (2.1) in which $a_{n}=c_{n}$ and $\theta=1 / 2$. The first class takes the form that

$g_{2 n}(t)=\left\{\begin{array}{ll}2 n t+b_{n}, & t \in\left[0, \frac{1}{2}\right), \\ 2 n t+d_{n}, & t \in\left[\frac{1}{2}, 1\right],\end{array} \quad\right.$ and $g_{2 n+1}(t)= \begin{cases}2 n t+b_{n}, & t \in\left[0, \frac{1}{2}\right), \\ 2 n t+d_{n}+\frac{1}{2}, & t \in\left[\frac{1}{2}, 1\right] .\end{cases}$

Our first theorem ensures that the set of functions $g_{n}, n \in Z$, is a spectral sequence.

Theorem 2.2. If $g_{n}, n \in Z$ is defined by (2.2), then it is a spectral sequence of $I$.

Proof. We prove that the corresponding system $f_{n}, n \in Z$, is an orthonormal basis for $L^{2}(I)$. The orthonormality of the functions $f_{n}, n \in Z$, follows directly from $I(2 n, 0)=0$ and $I(2 n, 1 / 2)=0$ for each $n \in Z \backslash\{0\}$, which are ensured by Lemma 2.1 .

It remains to prove the completeness of the system. To do this, we assume that $f \in L^{2}(I)$ such that

$$
\left\langle f, f_{n}\right\rangle=0, \text { for all } n \in Z,
$$


and we wish to show that $f=0$. Using assumption (2.3) and the definition of $g_{n}$, it can be verified that $\int_{0}^{\frac{1}{2}} f(t) e^{-2 \pi i(2 n t)} d t=0$ and $\int_{\frac{1}{2}}^{1} f(t) e^{-2 \pi i(2 n t)} d t=0$. By changes of variables, we obtain that for each $n \in Z$,

$$
\int_{0}^{1} f\left(\frac{t}{2}\right) e^{-2 \pi i n t} d t=0 \text { and } \int_{1}^{2} f\left(\frac{t}{2}\right) e^{-2 \pi i n t} d t=0 .
$$

Since the system $e_{n}, n \in Z$, forms an orthonormal basis for both spaces $L^{2}(I)$ and $L^{2}[1,2]$, it follows from (2.4) that $f=0$.

Next, we study systems $f_{n}, n \in Z$, associated with a different choice of exponents $g_{n}$. Specifically, for $a_{n}, b_{n} \in R$ and for a fixed $c \in R$, we set

$$
g_{2 n}(t):=2 n t+a_{n} \text { and } g_{2 n+1}(t)= \begin{cases}(2 n+c) t+b_{n}, & t \in\left[0, \frac{1}{2}\right), \\ (2 n+c) t+b_{n}+\frac{1-c}{2}, & t \in\left[\frac{1}{2}, 1\right] .\end{cases}
$$

The next theorem ensures that $g_{n}, n \in Z$, defined by (2.5) is a spectral sequence of $I$.

Theorem 2.3. If $g_{n}, n \in Z$, is defined by (2.5), then it is a spectral sequence of $I$.

Proof. Again, by using Lemma 2.1, it can be easily verified that the functions $f_{n}$ associated with the exponents $g_{n}$ defined by equations 2.5) are orthonormal on interval $I$.

To prove the completeness of the system, we let $f \in L^{2}(I)$ satisfy (2.3). A simple computation leads to the equation $\left\langle f, f_{2 n}\right\rangle=e^{-2 \pi i a_{n}}\left\langle f, e_{2 n}\right\rangle$. Now let $c_{n}$ denote the Fourier coefficients of function $f$, i.e., $c_{n}:=\left\langle f, e_{n}\right\rangle, n \in Z$. Thus, (2.3) and the equation above imply that $c_{2 n}=0$ for all $n \in Z$. The Fourier series of $f$ then becomes $f=\sum_{n \in Z} c_{2 n+1} e_{2 n+1}$, which, with the well-known Carleson theorem, implies that

$$
f\left(t+\frac{1}{2}\right)=\sum_{n \in Z} c_{2 n+1} e^{-2 \pi i(2 n+1)\left(t+\frac{1}{2}\right)}
$$

for almost every $t \in I$. Using the simple fact that $e^{-i \pi(2 n+1)}=-1$, we derive from the last formula that for almost every $t \in I$

$$
f\left(t+\frac{1}{2}\right)=-f(t)
$$

On the other hand, by the definition of $g_{2 n+1}$ we conclude that

$$
\left\langle f, f_{2 n+1}\right\rangle=\int_{0}^{\frac{1}{2}} f(t) e^{-2 \pi i\left[(2 n+c) t+b_{n}\right]} d t+\int_{\frac{1}{2}}^{1} f(t) e^{-2 \pi i\left[(2 n+c) t+b_{n}+\frac{1-c}{2}\right]} d t .
$$

In the second term, we replace variable $t$ by $t+1 / 2$ and it reduces to

$$
\begin{aligned}
\int_{0}^{\frac{1}{2}} f & \left(t+\frac{1}{2}\right) e^{-2 \pi i\left[(2 n+c) t+\frac{2 n+c}{2}+b_{n}+\frac{1-c}{2}\right]} d t \\
= & -e^{-2 \pi i b_{n}} \int_{0}^{\frac{1}{2}} f\left(t+\frac{1}{2}\right) e^{-2 \pi i(2 n+c) t} d t .
\end{aligned}
$$

Substituting equations (2.6) and (2.8) into (2.7) we conclude that $\left\langle f, f_{2 n+1}\right\rangle=$ $2 e^{-2 \pi i b_{n}} \int_{0}^{\frac{1}{2}} f(t) e^{-2 \pi i(2 n+c) t} d t$. It follows from this equation and (2.3) that

$$
\int_{0}^{1} f\left(\frac{t}{2}\right) e^{-2 \pi i\left(n+\frac{c}{2}\right) t} d t=0 .
$$


We introduce a new function defined by $\tilde{f}(t):=f\left(\frac{t}{2}\right) e^{-2 \pi i \frac{c}{2} t}, t \in I$, and conclude from the completeness of the classical Fourier basis and from the fact that $\tilde{f} \in L^{2}(I)$ that $\tilde{f}=0$ on $I$. Thus, $f=0$ on $\left[0, \frac{1}{2}\right]$. Again, by (2.6), we have $f=0$ on $I$. This completes the proof.

We remark that these two classes of bases include the classical Fourier basis as a special case. In fact, if we choose $a_{n}=b_{n}=0$ and $c=1$ in (2.5), then the classical Fourier basis $e_{n}$ is recovered.

To close this section, we consider the construction of real trigonometric bases with non-constant frequency functions. The classical Fourier basis $e_{n}, n \in Z$, has its real trigonometric polynomial bases for $L^{2}(I)$. We are interested in the question of whether the two families of exponential bases constructed in this section have their real trigonometric counterparts which form bases for space $L^{2}(I)$. We shall show that the real trigonometric form of the first family forms a basis for $L^{2}(I)$ but that of the second family does not in general. Given a sequence of functions $g_{n}$, $n \in Z$, we define their real trigonometric form $T_{n}$ by

$$
\begin{aligned}
& T_{0}(t):=1, \quad T_{2 n-1}(t):=\sqrt{2} \sin 2 \pi g_{n}(t), \\
& T_{2 n}(t):=\sqrt{2} \cos 2 \pi g_{n}(t), \quad n \in \mathbb{N}:=\{1,2, \ldots\} .
\end{aligned}
$$

Theorem 2.4. Let $g_{n}, n \in Z$, be defined by (2.2). Then, the system $T_{n}, n \in \mathbb{N}_{0}:=$ $\mathbb{N} \cup\{0\}$, defined by (2.9), forms an orthonormal basis for $L^{2}(I)$.

Proof. Using the definition of the functions $g_{n}$, it is easy to verify that for each $n \in \mathbb{N}_{0}, T_{n}$ is normal. The orthogonality of $T_{n}$ follows from the fact that for any $c, d \in R$ and $n \in \mathbb{N}$ the following equations hold:

$$
\int_{c}^{c+\frac{1}{2}} \cos 2 \pi(2 n t+d) d t=0
$$

and

$$
\int_{c}^{c+\frac{1}{2}} \sin 2 \pi(2 n t+d) d t=0
$$

To show the completeness of $T_{n}, n \in \mathbb{N}_{0}$, we assume that $\int_{0}^{1} f(t) \cos 2 \pi g_{2 n}(t) d t=$ 0 and $\int_{0}^{1} f(t) \cos 2 \pi g_{2 n+1}(t) d t=0$ for $f \in L^{2}(I)$. These with the definition of $g_{n}$ imply that

$$
\int_{0}^{1 / 2} f(t) \cos 2 \pi\left(2 n t+b_{n}\right) d t=\int_{1 / 2}^{1} f(t) \cos 2 \pi\left(2 n t+d_{n}\right) d t=0 .
$$

Likewise, equations $\int_{0}^{1} f(t) \sin 2 \pi g_{2 n}(t) d t=0$ and $\int_{0}^{1} f(t) \sin 2 \pi i g_{2 n+1}(t) d t=0$ imply that

$$
\int_{0}^{1 / 2} f(t) \sin 2 \pi\left(2 n t+b_{n}\right) d t=\int_{1 / 2}^{1} f(t) \sin 2 \pi\left(2 n t+d_{n}\right) d t=0 .
$$

On the other hand, we note that the classical system $1, \cos 2 \pi n t, \sin 2 \pi n t, n \in \mathbb{N}$, is complete in the space $L^{2}[\alpha, \alpha+1]$ for any $\alpha \in R$. Hence, system $1, \cos 4 \pi n t, \sin 4 \pi n t$, $n \in \mathbb{N}$, is complete in the space $L^{2}[\alpha, \alpha+1 / 2]$. Furthermore since for each $\beta_{n} \in R$ and $n \in Z$, there hold the formulas

$$
\cos 4 \pi n t=\cos \left(2 \pi \beta_{n}\right) \cos 2 \pi\left(2 n t+\beta_{n}\right)+\sin \left(2 \pi \beta_{n}\right) \sin 2 \pi\left(2 n t+\beta_{n}\right)
$$


and

$$
\sin 4 \pi n t=\cos \left(2 \pi \beta_{n}\right) \sin 2 \pi\left(2 n t+\beta_{n}\right)-\sin \left(2 \pi \beta_{n}\right) \cos 2 \pi\left(2 n t+\beta_{n}\right) .
$$

We conclude that $1, \cos 2 \pi\left(2 n t+\beta_{n}\right), \sin 2 \pi\left(2 n t+\beta_{n}\right), n \in \mathbb{N}$, is complete in the space $L^{2}[\alpha, \alpha+1 / 2]$. Therefore, by this argument with (2.10) and (2.11), we find that $f=0$ on both $[0,1 / 2]$ and $[1 / 2,1]$. Finally, we conclude that $f=0$ and the completeness is proved.

In the next theorem, we show that if the trigonometric system $T_{n}, n \in \mathbb{N}_{0}$, is constructed in terms of the frequency functions $g_{n}$ defined by (2.5), the system is not orthogonal in general.

Theorem 2.5. Let $g_{n}, n \in \mathbb{N}$, be the sequence of functions defined by (2.5)). Then, the system $T_{n}, n \in \mathbb{N}_{0}$, is not orthogonal unless $c \in Z$.

Proof. It suffices to show that $T_{n}$ with $g_{2 n+1}, n \in \mathbb{N}_{0}$ is not orthogonal unless $c \in Z$. For $n, m \in \mathbb{N}$ with $n \neq m$, it can be verified that $\left\langle\cos 2 \pi g_{2 n+1}, \cos 2 \pi g_{2 m+1}\right\rangle=0$ only if $c \in Z$.

\section{Characterizations}

In the last section, we present two constructions of piecewise linear spectral sequences $g_{n}, n \in Z$, with the knot at the half. Our next task is to investigate necessary and sufficient conditions on such a piecewise linear spectral sequence. We need the following technical lemma for the proof of necessary conditions.

Lemma 3.1. Suppose that $g_{n}, n \in Z$, defined by 2.1. with $a_{n}, c_{n} \in 2 Z$ and $\theta=1 / 2$ is a spectral sequence. For each $p \in 2 Z$, let $\mathcal{A}_{p}:=\left\{n \in Z: a_{n}=p\right\}$ and $\mathcal{C}_{p}:=\left\{n \in Z: c_{n}=p\right\}$. Then, the cardinality $\# \mathcal{A}_{p}=\# \mathcal{C}_{p}=2$.

Proof. By hypotheses, $f_{n}, n \in Z$, is an orthonormal basis for $L^{2}(I)$. We then have the Parseval identity, i.e., for any $f \in L^{2}(I)$,

$$
\sum_{n \in Z}\left|\int_{0}^{1} f(t) e^{2 \pi i g_{n}(t)} d t\right|^{2}=\int_{0}^{1}|f(t)|^{2} d t .
$$

In particular, for any $p \in 2 Z$, we choose $f(t):=e^{2 \pi i p t} \chi_{\left[0, \frac{1}{2}\right]}(t), t \in I$, where $\chi_{A}$ denotes the characteristic function of set $A$ and identity (3.1) reduces to

$$
\sum_{n \in Z}\left|\int_{0}^{\frac{1}{2}} e^{2 \pi i\left(a_{n}-p\right) t} d t\right|^{2}=\frac{1}{2} .
$$

Noting that $a_{n} \in 2 Z, p \in 2 Z$ and $a_{n}-p \in 2 Z$, by using Lemma 2.1, we conclude that

$$
\int_{0}^{\frac{1}{2}} e^{2 \pi i\left(a_{n}-p\right) t} d t= \begin{cases}0, & a_{n} \neq p \\ \frac{1}{2}, & a_{n}=p\end{cases}
$$

It follows from (3.2) and (3.3) that $\# \mathcal{A}_{p}=2$.

Likewise, if for any $p \in 2 Z$ we choose $f(t):=e^{2 \pi i p t} \chi_{\left[\frac{1}{2}, 1\right]}(t), t \in I$, in (3.1), we conclude that $\# \mathcal{C}_{p}=2$. 
We now consider our first case and set

$$
g_{n}(t):= \begin{cases}a_{n} t+b_{n}, & t \in\left[0, \frac{1}{2}\right), \\ a_{n} t+d_{n}, & t \in\left[\frac{1}{2}, 1\right],\end{cases}
$$

where $a_{n}, b_{n}, d_{n} \in R$. Note that given real-valued functions $g_{n}$, the system $e^{2 \pi i g_{n}}$, $n \in Z$, constitutes an orthonormal basis of $L^{2}(I)$ if and only if $e^{2 \pi i\left(g_{n}-g_{0}\right)}, n \in Z$, does. Without loss of generality, we will assume $g_{0}=0$ in our discussion.

The following theorem shows that the two constructions presented in Section 2 are essentially all that we can have, if we assume that $g_{n}$ has the form given in (3.4).

Theorem 3.2. Suppose that $g_{n}, n \in Z$, are defined by (3.4) and let $G:=\left\{g_{n}: n \in\right.$ $Z\}$. Then, $g_{n}, n \in Z$, is a spectral sequence of $I$ if and only if $G=\left\{u_{n}, v_{n}: n \in Z\right\}$, where $u_{n}$ and $v_{n}, n \in Z$, are defined by

$$
u_{n}(t):=\left\{\begin{array}{ll}
2 n t+b_{n}, & t \in\left[0, \frac{1}{2}\right), \\
2 n t+d_{n}, & t \in\left[\frac{1}{2}, 1\right],
\end{array} \quad \text { and } v_{n}(t):= \begin{cases}(2 n+c) t+b_{n}^{\prime}, & t \in\left[0, \frac{1}{2}\right), \\
(2 n+c) t+d_{n}^{\prime}, & t \in\left[\frac{1}{2}, 1\right],\end{cases}\right.
$$

with $b_{n}, d_{n}, b_{n}^{\prime}, d_{n}^{\prime}$ and $c$ satisfying one of the following conditions:

(i) $\left(b_{n}-b_{n}^{\prime}\right)-\left(d_{n}-d_{n}^{\prime}\right) \in Z+\frac{1}{2}$ and $c=0$.

(ii) $b_{n}-d_{n} \in Z, b_{n}^{\prime}-d_{n}^{\prime} \in Z+\frac{1-c}{2}$, and $c \in R \backslash 2 Z$.

Proof. The sufficient condition is proved by a similar argument used in the proof of Theorems 2.2 and 2.3 .

We now prove the necessary condition. Because the system $f_{n}, n \in Z$, is an orthonormal basis for $L^{2}(I)$ and $g_{0}=0$, we have that $\left\langle f_{n}, f_{0}\right\rangle=0$, for $n \in Z \backslash\{0\}$. Using (3.4), we conclude for $n \in Z \backslash\{0\}$ that $\int_{0}^{\frac{1}{2}} e^{2 \pi i\left(a_{n} t+b_{n}\right)} d t+\int_{\frac{1}{2}}^{1} e^{2 \pi i\left(a_{n} t+d_{n}\right)} d t=$ 0 . Thus, $\left(e^{2 \pi i b_{n}}+e^{2 \pi i\left(\frac{a_{n}}{2}+d_{n}\right)}\right) I\left(a_{n}, 0\right)=0$. This implies that either $I\left(a_{n}, 0\right)=0$, which by Lemma 2.1 is equivalent to $a_{n} \in 2 Z \backslash\{0\}$, or $e^{2 \pi i b_{n}}+e^{2 \pi i\left(\frac{a_{n}}{2}+d_{n}\right)}=0$, which is equivalent to $d_{n} \in Z-\frac{a_{n}}{2}+b_{n}+\frac{1}{2}$.

Associated with sequence $a_{n}$, we introduce two sets of exponent functions $G_{1}=$ : $\left\{g_{n} \in G: a_{n} \in 2 Z\right\}$ and $G_{2}:=\left\{g_{n} \in G: a_{n} \notin 2 Z, d_{n} \in Z-\frac{a_{n}}{2}+b_{n}+\frac{1}{2}\right\}$. We observe that $G=G_{1} \cup G_{2}$ and $G_{1} \neq \emptyset$ due to $g_{0} \in G_{1}$.

If $G_{2}=\emptyset$, then $G=G_{1}$ and by Lemma 3.1, for each $p \in 2 Z$, \# $\mathcal{A}_{p}=2$. In this case, we define the set $G$ by (3.5) with $c=0$. By the hypothesis of this theorem, we have that for $n \in Z,\left\langle e^{2 \pi i u_{n}(\cdot)}, e^{2 \pi i v_{n}(\cdot)}\right\rangle=0$. On the other hand, by using (3.5) with $c=0$, a direct computation leads to $\left\langle e^{2 \pi i u_{n}(\cdot)}, e^{2 \pi i v_{n}(\cdot)}\right\rangle=$ $\frac{1}{2}\left[e^{2 \pi i\left(b_{n}-b_{n}^{\prime}\right)}+e^{2 \pi i\left(d_{n}-d_{n}^{\prime}\right)}\right]$. Hence, $e^{2 \pi i\left(b_{n}-b_{n}^{\prime}\right)}+e^{2 \pi i\left(d_{n}-d_{n}^{\prime}\right)}=0$, and thus we have that $\left(b_{n}-b_{n}^{\prime}\right)-\left(d_{n}-d_{n}^{\prime}\right) \in Z+\frac{1}{2}$, which is condition (i).

If $G_{2} \neq \emptyset$, fixed $g_{n} \in G_{2}$, then for each $g_{m} \in G_{1}$, we have that $a_{m} \in 2 Z, a_{n} \notin 2 Z$ and $d_{n} \in Z-\frac{a_{n}}{2}+b_{n}+\frac{1}{2}$. Furthermore we have

$\int_{0}^{1} e^{2 \pi i\left(g_{m}(t)-g_{n}(t)\right)} d t=\int_{0}^{\frac{1}{2}} e^{2 \pi i\left[\left(a_{m}-a_{n}\right) t+\left(b_{m}-b_{n}\right)\right]} d t+\int_{\frac{1}{2}}^{1} e^{2 \pi i\left[\left(a_{m}-a_{n}\right) t+\left(d_{m}-d_{n}\right)\right]} d t$.

Since $a_{m} \in 2 Z$ and $d_{n} \in Z-\frac{a_{n}}{2}+b_{n}+\frac{1}{2}$, the second term in the right-hand side of (3.6) becomes

$$
\int_{0}^{\frac{1}{2}} e^{2 \pi i\left[\left(a_{m}-a_{n}\right) t+\frac{a_{m}-a_{n}}{2}+\left(d_{m}-d_{n}\right)\right]} d t=-e^{2 \pi i\left(d_{m}-b_{n}\right)} I\left(a_{m}-a_{n}, 0\right) .
$$


Hence, it follows from formula (3.6) and the hypothesis for the spectral sequence that $\left[e^{2 \pi i\left(b_{m}-b_{n}\right)}-e^{2 \pi i\left(d_{m}-b_{n}\right)}\right] I\left(a_{m}-a_{n}, 0\right)=0$.

Again, since $a_{m} \in 2 Z, a_{n} \notin 2 Z$, we have that $a_{m}-a_{n} \notin 2 Z$ and thus, by Lemma $2.1, I\left(a_{m}-a_{n}, 0\right) \neq 0$. Thus, $e^{2 \pi i b_{m}}=e^{2 \pi i d_{m}}$. This is equivalent to $b_{m}-d_{m} \in Z$ for each $g_{m} \in G_{1}$. By the definition of $G_{1}$, we have that $G_{1}=\left\{g_{n}: a_{n} \in 2 Z, b_{n}-d_{n} \in\right.$ $Z\}$. It follows from this equation that for each $n \in Z, e^{2 \pi i(2 n+1) t}$ is orthogonal to $e^{2 \pi i g_{m}(t)}$ for every $g_{m} \in G_{1}$. Hence, we conclude that $G_{2}$ contains infinitely many elements. Now for fixed $g_{l} \in G_{2}$ and for every $g_{n} \in G_{2}$ with $n \neq l$, because $d_{n} \in Z-\frac{a_{n}}{2}+b_{n}+\frac{1}{2}$ and $d_{l} \in Z-\frac{a_{l}}{2}+b_{l}+\frac{1}{2}$, the orthogonality of $f_{n}$ and $f_{l}$ implies $2 e^{2 \pi i\left(b_{n}-b_{l}\right)} I\left(a_{n}-a_{l}, 0\right)=0$. This ensures that $I\left(a_{n}-a_{l}, 0\right)=0$, which, by Lemma 2.1, is equivalent to $a_{n}-a_{l} \in 2 Z \backslash\{0\}$. Consequently, $a_{n} \in 2 Z+c$ with $c=: a_{l} \in R \backslash 2 Z$ for each $n \in Z_{2}$. Recalling the definition of $G_{2}$, we obtain that

$$
G_{2}:=\left\{g_{n} \in G: a_{n} \in 2 Z+c, d_{n} \in Z+b_{n}+\frac{1-c}{2}\right\} .
$$

The desired condition (ii) follows from (3.8) and (3.9).

In previous cases, the functions $g_{n}$ are defined in the way that $a_{n}=c_{n}$. It is easy to construct examples in which $a_{n} \neq c_{n}$. Note that for any real valued function $\tilde{g}$, $g_{n}+\tilde{g}, n \in Z$, is a spectral sequence of $I$ if and only if $g_{n}, n \in Z$, is. For example, if we choose

$$
\tilde{g}(t)= \begin{cases}a t, & t \in\left[0, \frac{1}{2}\right), \\ (2+c) t, & t \in\left[\frac{1}{2}, 1\right],\end{cases}
$$

with those $g_{n}$ constructed in the last two sections, we can construct many orthonormal bases $f_{n}, n \in Z$, for $L^{2}(I)$. To study this general case, we consider the form

$$
g_{n}(t)= \begin{cases}a_{n} t+b_{n}, & t \in\left[0, \frac{1}{2}\right), \\ c_{n} t+d_{n}, & t \in\left[\frac{1}{2}, 1\right] .\end{cases}
$$

Theorem 3.3. Suppose that $g_{n}, n \in Z$, is defined by (3.8) with $g_{0}=0, a_{1}=0$, and let $G:=\left\{g_{n}: n \in Z\right\}$. Then $g_{n}, n \in Z$, is a spectral sequence of $I$ if and only if $G=\left\{u_{n}, v_{n}: n \in Z\right\}$, where $u_{n}, v_{n}$ are defined by

$$
u_{n}(t)=\left\{\begin{array}{ll}
2 n t+b_{n}, & t \in\left[0, \frac{1}{2}\right), \\
c_{n} t+d_{n}, & t \in\left[\frac{1}{2}, 1\right],
\end{array} \quad v_{n}(t)= \begin{cases}2 n t+b_{n}^{\prime}, & t \in\left[0, \frac{1}{2}\right), \\
c_{n} t+d_{n}^{\prime}, & t \in\left[\frac{1}{2}, 1\right],\end{cases}\right.
$$

with constants $b_{n}, c_{n}, d_{n}, b_{n}^{\prime}$ and $d_{n}^{\prime}$ satisfying the conditions that $\left\{c_{n}: n \in Z\right\}=$ $2 Z, c_{n} \neq c_{m}$ for $n \neq m$ and $\left(b_{n}^{\prime}-d_{n}^{\prime}\right)-\left(b_{n}-d_{n}\right) \in Z+\frac{1}{2}$.

Proof. We first prove the sufficiency. Due to (3.9) the orthogonality of $e^{2 \pi i u_{n}}$, $e^{2 \pi i v_{n}}, n \in Z$, follows from Lemma 2.1 with the assumption $\left\{c_{n}: n \in Z\right\}=2 Z$, $c_{n} \neq c_{m}$ for $n \neq m$. The proof of the completeness for the system is similar to Theorem 2.2. Suppose that $\left\langle f, e^{2 \pi i u_{n}}\right\rangle=\left\langle f, e^{2 \pi i v_{n}}\right\rangle=0, n \in Z$, and we wish to derive $f=0$. In fact, by (3.9), we have that $e^{2 \pi i b_{n}} A_{n}+e^{2 \pi i d_{n}} B_{n}=0$ and $e^{2 \pi i b_{n}^{\prime}} A_{n}+$ $e^{2 \pi i d_{n}^{\prime}} B_{n}=0$, where $A_{n}:=\int_{0}^{\frac{1}{2}} f(t) e^{-2 \pi i 2 n t} d t$ and $B_{n}:=\int_{\frac{1}{2}}^{1} f(t) e^{-2 \pi i c_{n} t} d t$. Because of the given assumption $\left(b_{n}^{\prime}-d_{n}^{\prime}\right)-\left(b_{n}-d_{n}\right) \in Z+\frac{1}{2}$, the second equation reduces to $e^{2 \pi i\left[d_{n}^{\prime}+\left(b_{n}-d_{n}\right)+\frac{1}{2}\right]} A_{n}+e^{2 \pi i d_{n}^{\prime}} B_{n}=0$ or equivalently, $-e^{2 \pi i b_{n}} A_{n}+$ $e^{2 \pi i d_{n}^{\prime}} B_{n}=0$. Combining this with the first equation, we obtain that $A_{n}=B_{n}=0$. Since $\left\{c_{n}: n \in Z\right\}=2 Z$, we conclude that $f=0$.

Now, we prove the necessity. Since $f_{n}, n \in Z$, is an orthonormal basis of $L^{2}(I)$, for $f \in L^{2}(I)$ we have the Parseval identity (3.1). In particular, we choose 
$f=\chi_{\left[0, \frac{1}{2}\right]}$, the characteristic function on the interval $\left[0, \frac{1}{2}\right]$ in (3.1). Note that the hypotheses $g_{0}=0$ and $a_{1}=0$ imply $\left|\int_{0}^{\frac{1}{2}} e^{2 \pi i\left(a_{0} t+b_{0}\right)} d t\right|=\frac{1}{2}$ and $\left|\int_{0}^{\frac{1}{2}} e^{2 \pi i\left(a_{1} t+b_{1}\right)} d t\right|$ $=\frac{1}{2}$. Hence, in this case, identity (3.1) reduces to $\sum_{n \in Z, n \neq 0,1}\left|\int_{0}^{\frac{1}{2}} e^{2 \pi i\left(a_{n} t+b_{n}\right)}\right|^{2}=$ 0 . That is, $I\left(a_{n}, 0\right)=0$, for $n \in Z \backslash\{0,1\}$. It follows from Lemma 2.1 that $a_{n} \in 2 Z \backslash\{0\}$ for $n \in Z \backslash\{0,1\}$. By $g_{0}=0$, the last equation and the orthogonality of $f_{n}, n \in Z$, we obtain that

$$
\begin{aligned}
\left\langle e^{2 \pi i g_{n}(t)}, e^{2 \pi i g_{0}(t)}\right\rangle & =\int_{0}^{\frac{1}{2}} e^{2 \pi i\left(a_{n} t+b_{n}\right)} d t+\int_{\frac{1}{2}}^{1} e^{2 \pi i\left(c_{n} t+d_{n}\right)} d t \\
& =\int_{\frac{1}{2}}^{1} e^{2 \pi i\left(c_{n} t+d_{n}\right)} d t=0 .
\end{aligned}
$$

Furthermore, we have that $I\left(c_{n}, 1 / 2\right)=0$, for $n \in Z \backslash\{0,1\}$. Again, by Lemma 2.1 , this implies that $c_{n} \in 2 Z \backslash\{0\}$ for $n \in Z \backslash\{0,1\}$.

Next we prove $c_{1} \in 2 Z$. Since $a_{3} \in 2 Z \backslash\{0\}$ and $a_{1}=0$, we have that

$$
\int_{0}^{\frac{1}{2}} e^{2 \pi i\left[\left(a_{3}-a_{1}\right) t+\left(b_{3}-b_{1}\right)\right]} d t=0
$$

by Lemma 2.1. Using the orthogonality of $f_{n}, n \in Z$, we conclude that

$$
\int_{\frac{1}{2}}^{1} e^{2 \pi i\left[\left(c_{3}-c_{1}\right) t+\left(d_{3}-d_{1}\right)\right]} d t=0 .
$$

This is equivalent to $c_{3}-c_{1} \in 2 Z \backslash\{0\}$, due to Lemma 2.1. Moreover, since $c_{3} \in 2 Z$, we have that $c_{1} \in 2 Z$.

Summarizing the discussion above with the assumption that $g_{0}=0$, we conclude that $a_{n}, c_{n} \in 2 Z$, for $n \in Z$. Moreover, it follows from Lemma 3.1 that $\# \mathcal{A}_{p}=$ $\# \mathcal{C}_{p}=2$, for each $p \in 2 Z$. Therefore, we can assume that $G=\left\{u_{n}, v_{n}: n \in Z\right\}$, where $u_{n}$ is as defined in (3.9) and $v_{n}$ is as defined in (3.9) with $c_{n}$ replaced by $c_{n}^{\prime}$ and with $c_{n}, c_{n}^{\prime} \in 2 Z$. Note that $c_{n}-c_{n}^{\prime} \in 2 Z$ and

$$
I\left(c_{n}-c_{n}^{\prime}, 1 / 2\right)= \begin{cases}\frac{1}{2}, & c_{n}=c_{n}^{\prime}, \\ 0, & c_{n} \neq c_{n}^{\prime} .\end{cases}
$$

Again, by the orthogonality of $f_{n}, n \in Z$, we obtain that

$$
\left\langle e^{2 \pi i u_{n}}, e^{2 \pi i v_{n}}\right\rangle=\frac{1}{2} e^{2 \pi i\left(b_{n}-b_{n}^{\prime}\right)}+e^{2 \pi i\left(d_{n}-d_{n}^{\prime}\right)} I\left(c_{n}-c_{n}^{\prime}, 1 / 2\right)=0 .
$$

Consequently, we must have that $I\left(c_{n}-c_{n}^{\prime}, 1 / 2\right) \neq 0$. Employing (3.10) we find that $c_{n}=c_{n}^{\prime}$. In this case, equation (3.11) implies that $e^{2 \pi i\left(b_{n}-b_{n}^{\prime}\right)}+e^{2 \pi i\left(d_{n}-d_{n}^{\prime}\right)}=0$ and hence, $\left(b_{n}^{\prime}-d_{n}^{\prime}\right)-\left(b_{n}-d_{n}\right) \in Z+\frac{1}{2}$.

For $n \neq m$, by assumption, $\left\langle e^{2 \pi i u_{n}}, e^{2 \pi i u_{m}}\right\rangle=0$. Using the definition of function $u_{n}$, we have that

$$
\left\langle e^{2 \pi i u_{n}}, e^{2 \pi i u_{m}}\right\rangle=\int_{0}^{\frac{1}{2}} e^{2 \pi i\left[(2 n-2 m) t+\left(b_{n}-b_{m}\right)\right]}+\int_{\frac{1}{2}}^{1} e^{2 \pi i\left[\left(c_{n}-c_{m}\right) t+\left(d_{n}-d_{m}\right)\right]} .
$$

By Lemma 2.1, we see that the first integral in the right-hand side of the above equation vanishes. Therefore, by this equation and our assumption, we obtain that $e^{2 \pi i\left(d_{n}-d_{m}\right)} I\left(c_{n}-c_{m}, 1 / 2\right)=0$, from which we conclude that $c_{n} \neq c_{m}$ again by Lemma 2.1. 


\section{Non-CONTINUity OF SPECTRAL SEQUENCES}

We will prove in this section that the spectral sequences $g_{n}, n \in Z$, of form (2.1), cannot be continuous except for the classical case. We first develop a technical lemma.

Lemma 4.1. For $\theta \in(0,1)$ let

$$
g(t)= \begin{cases}a t+b, & t \in[0, \theta) \\ c t+d, & t \in[\theta, 1]\end{cases}
$$

be continuous and assume that $\int_{0}^{1} e^{2 \pi i g(t)} d t=0$. Then, either $a=c$ or $\theta a \in Z$, $(1-\theta) c \in Z$.

Proof. Note that the continuity of $g$ implies $d=(a-c) \theta+b$. Using this relation and the hypothesis of the lemma, we have that $\int_{0}^{\theta} e^{2 \pi i a t} d t+e^{2 \pi i(a-c) \theta} \int_{\theta}^{1} e^{2 \pi i c t} d t=0$.

We next show that if either $a$ or $c$ is zero, then both of them are zero. To this end, we suppose $a=0$ and $c \neq 0$. Integration of the equation above yields that

$$
\theta+e^{-2 \pi i c \theta} \frac{e^{2 \pi i c}-e^{2 \pi i c \theta}}{2 \pi i c}=\frac{1}{2 \pi i c}\left[2 \pi i c \theta+e^{2 \pi i(1-\theta) c}-1\right]=0 .
$$

However,

$$
\left|2 \pi i c \theta+e^{2 \pi i(1-\theta) c}-1\right| \geq|2 \pi i c \theta-1|-\left|e^{2 \pi i(1-\theta) c}\right|=\sqrt{1+(2 \pi c \theta)^{2}}-1>0,
$$

a contradiction. Likewise, $c=0$ implies $a=0$.

We next consider the case when $a \neq 0$ and $c \neq 0$. In this case, we have that

$$
\frac{e^{2 \pi i a \theta}-1}{2 \pi i a}+\frac{e^{2 \pi i(a-c) \theta}}{2 \pi i c}\left[e^{2 \pi i c}-e^{2 \pi i c \theta}\right]=0 .
$$

Multiplying both sides by $2 \pi i a c e^{-2 \pi i a \theta}$, we have that

$$
c\left(1-e^{-2 \pi i a \theta}\right)=a\left[1-e^{2 \pi i(1-\theta) c}\right],
$$

which is equivalent to

$$
c(1-\cos 2 \pi a \theta)=a[1-\cos 2 \pi(1-\theta) c]
$$

and

$$
c \sin 2 \pi a \theta=-a \sin 2 \pi(1-\theta) c .
$$

We conclude from (4.1) that $\theta a \in Z$ if and only if $(1-\theta) c \in Z$ and from (4.2) that $\theta a \in Z / 2$ if and only if $(1-\theta) c \in Z / 2$.

To complete the proof of this lemma, it suffices to prove $a=c$ under the assumptions $\theta a \notin Z$ or $(1-\theta) c \notin Z$. By the conclusion stated in the last paragraph we know that $\theta a \notin Z$ is equivalent to $(1-\theta) c \notin Z$. Hence, in the rest of this proof, we assume that $\theta a \notin Z$. If $\theta a \in \frac{Z}{2}$, then $\theta a \in \frac{Z}{2} \backslash Z$ and $(1-\theta) c \in \frac{Z}{2} \backslash Z$. That is, both $2 \theta a$ and $2(1-\theta) c$ are odd integers. Thus, by (4.1), $a=c$. If $\theta a \notin Z / 2$, then $(1-\theta) c \notin Z / 2$. Therefore, (4.1) and (4.2) imply

$$
\frac{c(1-\cos 2 \pi a \theta)}{c \sin 2 \pi a \theta}=\frac{a[1-\cos 2 \pi(1-\theta) c]}{-a \sin 2 \pi(1-\theta) c} .
$$

Now we have $\tan \pi a \theta=-\tan \pi(1-\theta) c$, which ensures that $\pi a \theta=-\pi(1-\theta) c+\pi k$ with $k \in Z$. Substituting it into (4.2), we conclude that $a=c$ because $(1-\theta) c \notin$ $Z / 2$. 
With this lemma, we are ready to show our main result in this section. To state the theorem, we define

$$
g_{n}(t):= \begin{cases}a_{n} t+b_{n}, & t \in[0, \theta) \\ c_{n} t+d_{n}, & t \in[\theta, 1]\end{cases}
$$

Theorem 4.2. Suppose that $g_{n}, n \in Z$, defined by (4.3) with $g_{0}=0$ is a continuous spectral sequence of $I$. Let $G:=\left\{g_{n}: n \in Z\right\}$. Then, $G=\left\{h_{n}: n \in Z\right\}$ where $h_{n}(t)=n t+b_{n}, t \in I, n \in Z$.

Proof. According to Lemma 4.1, either $a_{n}=c_{n}$ or $\theta a_{n} \in Z,(1-\theta) c_{n} \in Z$. If $a_{n}=c_{n}$, then due to the continuity of $g_{n}$ the corresponding $g_{n}(t)=a_{n} t+b_{n}, t \in I$. Now, since $g_{0}=0$ and for $n \neq 0,\left\langle e^{2 \pi i g_{n}}, e^{2 \pi i g_{0}}\right\rangle=\int_{0}^{1} e^{2 \pi i g_{n}(t)} d t=0$, we have that $a_{n} \in Z$. We denote by $Z_{1}$ the collection of all such $a_{n}$. Let $G_{1}:=\{h(t)=l t+e \in$ $\left.G: l \in Z_{1}\right\}$ and $G_{2}=G \backslash G_{1}$. Then, $G=G_{1} \cup G_{2}$. It suffices to show $G_{2}=\emptyset$, since in this case the completeness of $\left\{e^{2 \pi i h}: h \in G_{1}\right\}$ leads to $Z_{1}=Z$ and furthermore to the desired result.

Suppose $g \in G_{2}$; we would have a contradiction. To see this, we let

$$
g(t)= \begin{cases}a t+b, & t \in[0, \theta), \\ c t+d, & t \in[\theta, 1] .\end{cases}
$$

Then, by the definition of $G_{2}$ and the continuity of $g$, we see the properties that (i) $a \neq c$, (ii) $\theta a \in Z,(1-\theta) c \in Z$, and (iii) $d=(a-c) \theta+b$.

We next consider two cases.

Case 1: $c \in Z_{1}$. In this case, we let $h(t)=c t+e, t \in I$. Then, $h \in G_{1}$. By orthogonality, we have that

$$
\left\langle e^{2 \pi i g}, e^{2 \pi i h}\right\rangle=\int_{0}^{\theta} e^{2 \pi i[(a t+b)-(c t+e)]} d t+\int_{\theta}^{1} e^{2 \pi i[(c t+d)-(c t+e)]} d t .
$$

By a direct computation, equation (4.4) becomes

$$
e^{2 \pi i(b-e)} \frac{e^{2 \pi i(a-c) \theta}-1}{2 \pi i(a-c)}+(1-\theta) e^{2 \pi i(d-e)}=0 .
$$

Note that $\theta a \in Z$ implies $e^{2 \pi i a \theta}=1$ and property (iii) implies

$$
e^{2 \pi i(d-e)}=e^{2 \pi i[(a-c) \theta+(b-e)]} .
$$

By using properties (ii) and (iii), the above identity reduces to

$$
e^{2 \pi i(b-e)} e^{-2 \pi c \theta} \frac{1}{2 \pi i(a-c)}\left[1-e^{2 \pi i c \theta}+2 \pi i(1-\theta)(a-l c)\right]=0 .
$$

From this, we have arrived at $1-e^{2 \pi i c \theta}+2 \pi i(1-\theta)(a-c)=0$. However,

$$
\left|1-e^{2 \pi i c \theta}+2 \pi i(1-\theta)(a-c)\right| \geq|1+2 \pi i(1-\theta)(a-c)|-\left|e^{2 \pi i l \theta}\right|>0,
$$

a contradiction.

Case 2. $c \notin Z_{1}$. We first show the claim that if $l \in Z_{1}$, then $l \theta \in Z$. If $l=a$, then $l \theta=\theta a \in Z$ due to property (ii). If $l \neq a$, define $h(t)=l t+e, t \in I$. Note that $c \neq Z_{1}$. Then

$$
\int_{0}^{1} e^{2 \pi i[g(t)-h(t)]} d t=e^{2 \pi i(b-e)} \frac{e^{2 \pi i(a-l) \theta}-1}{2 \pi i(a-l)}+e^{2 \pi i(d-e)} \frac{e^{2 \pi i(c-l)}-e^{2 \pi i(c-l) \theta}}{2 \pi i(c-l)}=0,
$$


which by property (iii) reduces to

$$
\frac{e^{2 \pi i(a-l) \theta}-1}{2 \pi i(a-l)}+e^{2 \pi i(a-c) \theta} \frac{e^{2 \pi i(c-l)}-e^{2 \pi i(c-l) \theta}}{2 \pi i(c-l)}=0 .
$$

Finally, it follows from property (ii) that $\frac{e^{-2 \pi i l \theta}-1}{(a-l)}+\frac{1-e^{-2 \pi i l \theta}}{(c-l)}=0$. Since $a \neq c$, one receives $1-e^{-2 \pi i l \theta}=0$. That is, $l \theta \in Z$. Our claim is proved.

Now we are in the position to receive a contradiction. Let $h_{0}=\chi_{[0, \theta)}$. Then, for each $g \in G_{2}$, because of property (ii), we have that $\left\langle h_{0}, e^{2 \pi i g}\right\rangle=\int_{0}^{\theta} e^{2 \pi i(a t+b)} d t=$ $\theta \int_{0}^{1} e^{2 \pi i(a \theta t+b)} d t=0$. Similarly, for each $h \in G_{1}$, by our claim, $\left\langle h_{0}, e^{2 \pi i h}\right\rangle=$ $\int_{0}^{\theta} e^{2 \pi i(l t+b)} d t=\theta \int_{0}^{1} e^{2 \pi i(l \theta t+b)} d t=0$. But the completeness of $f_{n}, n \in Z$, implies $h_{0}=0$, which is a contradiction.

\section{A piecewise constant spectral sequence and the Walsh system}

In this section, we will construct a piecewise constant spectral sequence of $I$. From this sequence, we recover the classical Walsh system (cf., [6, 7] 8]).

Let $J_{n}=:\left\{0,1, \ldots, 2^{n}-1\right\}$. Then $J_{n} \subset J_{n+1}$ for $n \in \mathbb{N}_{0}$. We now describe the construction of the piecewise constant spectral sequence $g_{n}, n \in \mathbb{N}_{0}$. Beginning with $g_{0}=0$ on the interval $I$, we define for $j \in J_{n}$ recursively

$$
g_{2^{n}+j}(t)= \begin{cases}g_{j}(t), & t \in\left[t_{n, 2 k}, t_{n, 2 k+1}\right) \\ g_{j}(t)+\frac{1}{2}, & t \in\left[t_{n, 2 k+1}, t_{n, 2 k+2}\right)\end{cases}
$$

for $j \in J_{n}$, where $t_{n, k}:=\frac{k}{2^{n+1}}, k=0,1, \ldots, 2^{n+1}$. Clearly, $g_{j}$ is well defined for $j \in J_{n+1}$ and each $g_{n}, n \in \mathbb{N}_{0}$, is a piecewise constant function with the values in $Z \cup\left(Z+\frac{1}{2}\right)$.

Theorem 5.1. Let $g_{n}, n \in \mathbb{N}_{0}$, be given as above. Then, $g_{n}, n \in \mathbb{N}_{0}$, is a spectral sequence of $I$ and $f_{n}, n \in \mathbb{N}_{0}$, is the Walsh system on $I$.

Proof. We use the induction on $n$ to prove the orthogonality of system $f_{n}, n \in \mathbb{N}_{0}$, corresponding to $g_{n}, n \in \mathbb{N}_{0}$. That is, we will prove that $\left\langle e^{2 \pi i g_{j}}, e^{2 \pi i g_{l}}\right\rangle=0$, for $j, l \in J_{n}$.

We first consider the case $n=1$. Since $g_{0}=0$ and $g_{1}=\frac{1}{2} \chi_{\left[\frac{1}{2}, 1\right]}$, it is easy to see $\left\langle e^{2 \pi i g_{0}}, e^{2 \pi i g_{1}}\right\rangle=0$.

We assume that $\left\langle e^{2 \pi i g_{j}}, e^{2 \pi i g_{l}}\right\rangle=0$, for $j, l \in J_{n}$ and show that it holds for $j, l \in J_{n+1}$. Suppose that both $j$ and $l$ are in $J_{n+1} \backslash J_{n}$. Hence, there exist $j_{0}, l_{0} \in J_{n}$ such that $j=2^{n}+j_{0}$ and $l=2^{n}+l_{0}$. In this case, using the induction hypothesis, we have that $\left\langle e^{2 \pi i g_{j}}, e^{2 \pi i g_{l}}\right\rangle=\left\langle e^{2 \pi i g_{j_{0}}}, e^{2 \pi i g_{l_{0}}}\right\rangle=0$. Suppose that $j$ is in $J_{n+1} \backslash J_{n}$ and $l \in J_{n}$. Hence, there exists $j_{0} \in J_{n}$ such that $j=2^{n}+j_{0}$. Consequently, by the definition of $g_{2^{n}+j_{0}}$, we obtain that

$$
\begin{aligned}
& \left\langle e^{2 \pi i g_{j}}, e^{2 \pi i g_{l}}\right\rangle=\int_{0}^{1} e^{2 \pi i\left(g_{2}{ }^{n}+j_{0}-g_{l}\right)} d t \\
& =\sum_{k=0}^{2^{n}-1}\left[\int_{t_{n, 2 k}}^{t_{n, 2 k+1}} e^{2 \pi i\left(g_{j_{0}}-g_{l}\right)} d t+\int_{t_{n, 2 k+1}}^{t_{n, 2 k+2}} e^{2 \pi i\left(g_{j_{0}}-g_{l}+\frac{1}{2}\right)} d t\right] .
\end{aligned}
$$


Since on intervals $\left(t_{n, 2 k}, t_{n, 2 k+1}\right)$ and $\left(t_{n, 2 k+1}, t_{n, 2 k+2}\right), c_{j_{0}, l}:=g_{j_{0}}(t)-g_{l}(t)$ has the same constant value, we have that

$$
\left\langle e^{2 \pi i g_{j}}, e^{2 \pi i g_{l}}\right\rangle=\sum_{k=0}^{2^{n}-1} \frac{1}{2^{n+1}}\left(e^{2 \pi i c_{j_{0}, l}}-e^{2 \pi i c_{j_{0}, l}}\right)=0 .
$$

This advances the induction hypothesis and proves the orthogonality.

To show the completeness, we denote by $X_{n}$ the space of the piecewise constants with the knots at dyadic points $\frac{k}{2^{n}}, k=1,2, \ldots, 2^{n}-1$. Note that the dimension of $X_{n}$ is $2^{n}$. Also, $\left\{e^{2 \pi i g_{j}}: j \in J_{n}\right\} \subseteq X_{n}, \# J_{n}=2^{n}$ and $e^{2 \pi i g_{j}}, j \in J_{n}$, are orthonormal. We then conclude that $\left\{e^{2 \pi i g_{j}}: j \in J_{n}\right\}$ spans $X_{n}$. Since $\left\{X_{n}: n \in\right.$ $\left.\mathbb{N}_{0}\right\}$ is dense in $L^{2}(I)$, the completeness follows.

The identification of $f_{n}, n \in \mathbb{N}_{0}$, with the classical Walsh system on $I$ can be verified by an induction on $n$.

\section{REFERENCES}

[1] Q. Chen, N. Huang, S. Riemenschneider and Y. Xu, A B-spline approach for empirical mode decomposition, Adv. Comp. Math., to appear.

[2] I. Daubechies, Ten Lectures on Wavelets, CBMS 61, SIAM, Philadelphia, 1992. MR 1162107 (93e:42045)

[3] K. Grochenig, Foundations of Time-Frequency Analysis, Birkhauser, Boston, 2001. MF $1843717(2002 \mathrm{~h}: 42001)$

[4] N. E. Huang, Z. Shen, S. R. Long, M. C. Wu, H. H. Shih, Q. Sheng, N. Yen, C. C. Tung and H. H. Liu, The empirical mode decomposition and the Hilbert spectrum for nonlinear and non-stationary time series analysis, Proc. R. Soc. Land. A (1998) 454, 903-995. MR1631591 (99d:76082)

[5] J. C. Lagarias and Y. Wang, Spectral sets and factorizations of finite Abelian groups, J. Funct. Anal. 145 (1997), 73-98. MR.1442160 (98b:47011b)

[6] S. Mallat, A Wavelet Tour of Signal Processing, Academic Press, San Diego, 1998. MF 1614527 (99m:94012)

[7] C. A. Micchelli and Y. Xu, Using the matrix refinement equation for the construction of wavelets on invariant sets, Appl. Comput. Harmon. Anal. 1 (1994), 391-401. MR1310661 (96k:42044)

[8] R. E. Paley, A remarkable series of orthogonal functions I, Proc. Landon Math. Soc. 34 (1932), 241-279.

[9] Y. Wang, Wavelets, tiling and spectral sets, Duke Math. J., 114 (2002), no. 1, 43-57. MF, 1915035 (2003e:42057)

[10] R. Young, An Introduction to Non-harmonic Fourier Series, Academic Press, London, 1980. MF $0591684(81 \mathrm{~m}: 42027)$

Department of Applied Mathematics, Beijing Polytechnic University, Pingle Yuan 100, Beijing 100022, People's Republic of China

Department of Mathematics, 215 Carnegie Hall, Syracuse University, Syracuse, New York 13244 - And - Institute of Mathematics, ACAdemy of Mathematics and System Sciences, The Chinese Academy of Sciences, Beijing 100080, People's Republic of China 